\section{Целевой уровень артериального давления при лечении артериальной гипертензии у пациентов пожилого возраста с хронической сердечной недостаточностью}

M.C. ЧЕРНЯЕВА', O.А. ОСТРОУМОВА 2,3

ФГБУ АПО «Центраљьная государственная медииинская акаеемия» Управления Аелами Презимента Российской Фееераиии, Москва, Россия ФГБОУ ВО «РНИМУ им. Н.И. Пирогова» Минзарава России - ОСП «Российский геронтологический научно-киинический чентр», Москва, Россия; ${ }^{3}$ ФГАОУ ВО «Первый Московский госуарственный медицинский университет им. И.М. Сеченова» Минздрава России (Сеченовский университет), Москва, Россия

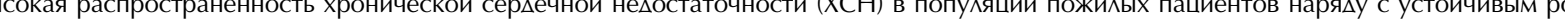
стом численноспи пожилого населения как в Рессии, такив западных странах все бокьше привлекает внимание врачей к проблеме, связанной с вееением Аанного заболевания. Известно, что ведушим фактором риска развития ХСН является повышенное артериальное Аавлени

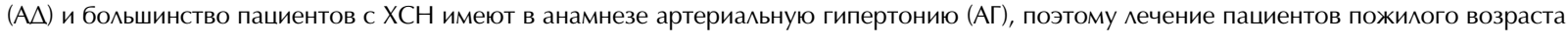
AГ и XCH явяяется одним из важных направеений в профииактике прогрессирования ХСН, Снижения количества госпитализаций и смерт ности. Аечение АГ у пожимых имеет свои особенности, связанные с функциональным статусом пашиентов и их способностью переносит

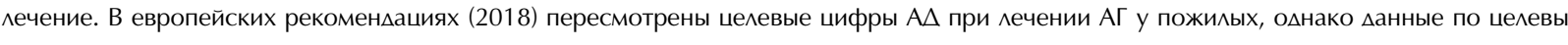

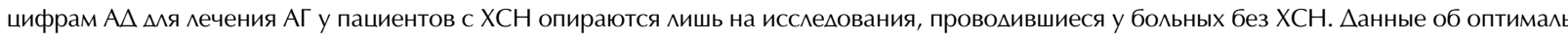
ном целевом уровне у пациентов С AГ и XCH преАставлены в еАиничных исслеАованиях. В настояшей статье проанализирована взаимосвяз уровня АА и сердечно-сосудистых событий и смертности отАельно Аяя пациентов С АГ и сердечной неАостаточностью с низкой фракцие выброса левого желудочка и с сохраненной фракцией выброса левого желудочка. Результаты многих исслеевваний показывают, что боле низкий уровень систояического $A \Delta(<120$ мм рт. ст.) и Аиастолического $А \triangle$ (<80 мм рт. ст.) ассоииирован с развитием неблагоприятны сердечно-сосудистых событий, особенно у пациентов с сердечной недостаточностью с низкой фракцией выброса левого желудочка.

Киючевые слова. Артериальная гипертензия, пожимой возраст, целевой уровень артериаљьного Аавления, хроническая сердечная не Аостаточность, сердечная неАостаточность с низкой фракцией выброса левого жеиудочка, сердечная нецостаточность с сохраненно фракцией выброса левого желуцочка.

\section{Target blood pressure levels in elderly patients with chronic heart failure}

M.S. CHERNIAEVA', O.D. OSTROUMOVA',3

Central state medical academy of department of presidential affairs, Moscow, Russian Federation; 2 Federal State Budgetary Educational Institution o Higher Education «N.I. Pirogov Russian National Research Medical University» of the Ministry of Health of the Russian Federation; Russian Clinical and Research Center of Gerontology, Moscow, Russian Federation; ${ }^{3}$ I.M. Sechenov First Moscow State Medical University, Moscow, Russian Federation

The high prevalence of chronic heart failure (CHF) in the elderly patients, along with the steady growth of the elderly population, both in Russia and in Western countries, is increasingly attracting the attention of doctors to the problem associated with the management of this disease. It is know that the leading risk factor for CHF is high blood pressure (BP) and most patients with CHF have a history of hypertension (H), so the treatment of elderly patients with $\mathrm{H}$ and $\mathrm{CHF}$ is the major focus in the slowing $\mathrm{CHF}$ progression, reducing the heart failure hospitalisation and mortality. Treatment of hypertension in the elderly has some specific features associated with the functional status of patients and their ability to tolerate treatment. The European recommendations (2018) revised target blood pressure levels in the elderly patients, however, data on target blood pressure levels in patients with CHF are based only on studies conducted in patients without CHF, data on the optimal target blood pressure levels in patients with hypertension and CHF are presented in single studies. In this article we analyze the relationship between blood pressure levels and cardiovascular events and mortality separately for patients with hypertension and heart failure with reduced ejection fraction and with preserved ejection fraction. Several studies show that lower systolic blood pressure $(<120 \mathrm{~mm} H \mathrm{Hg})$ and diastolic blood pressure $(<80 \mathrm{~mm} \mathrm{Ho})$ is associated with the increased risk of cardiovascular events, especially in patients with heart failure with reduced ejection fraction.

Key words. Arterial hypertension, older age, target blood pressure, chronic heart failure, heart failure with reduced ejection fraction, heart failure with preserved ejection fraction.

Свеления об авторах:

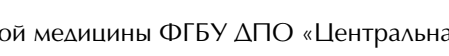

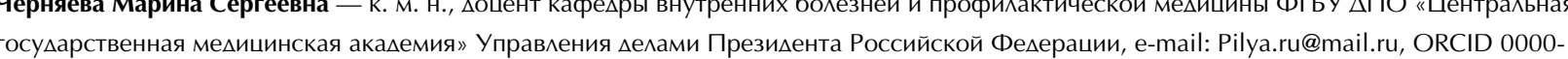
0003-3091-7904

Остроумова ОАьга Амитриевна - А. М. н, проф., зав. лабораторией кминической фармакомогии и фармакотерапии ФГБОУ ВО «РНИМУ им. Н.И. Пирогова» Минзарава России - ОСП «Российский геронтооггический научно-кминический центр», проф. кафедры кминической фармакооогии и пропееевтики внутренних болезней Первого Московского госуаАрственного медииинского университета им. И.М.
ва (Сеченовский университет). е-mail: ostroumova.olga@mail.ru, ORCID 0000-0002-0795-8225 (автор, ответственный за переписку)

Повышенное артериальное давление (АД) является ведущим фактором риска развития хронической сердечной недостаточности (ХСН) [1], а большинство пациенто с ХСН имеют в анамнезе артериальную гипертонию (АГ) [2]. АГ вызывает типертрофию левого желудочка (ГЛЖ), которая нарушает его расслабление (диастолическая дисфункция) и является мощным предиктором развития $\mathrm{XCH}$ с сохраненной фракцией выброса левого желудочка (СНсФВ) даже без предшествующего острого инфаркта миокарда [2]. Обусловленный АГ фиброз миокарда и сосудистой стенки, структурные изменения крупных и мелких артерий также вносят свой вклад в развитие ХСН [2]. Доказано, что лечение АГ оказывает значительное влияние на снижение риска развития новых случаев ХСН у пациентов с АГ [3-6], особенно у пожилых и очень пожилых пациентов [7-9]. Среди препаратов для лечения АГ, которые хорошо зарекомендовали себя в предопращении развития ХСН, выделяют диуретики, бета-адреноблокаторь ( $\beta$-АБ), ингибиторы ангиотензинпревращающего фермента (иАПФ) и блокаторы рецепторов к ангиотензину II (БРА), а также их комбинации [5, 10] (класс рекомендаций и уровень достоверности данных в российских клинических рекомендациях по диагностике, профилактике и лечению сердечной недостаточности 2018 г. - IA) [11], причем, по данным сравнительных исследований, блокаторы кальциевых каналов (БКК) оказались менее эффективными [12]. Недигидропиридиновые БКК противопоказаны при АГ в сочетании с ХСН [10]. Имеются сведения о большем риске прогрессирования ХСН при лечении больных АГ БКК дигидропиридинового ряда в сравнении с диуретиками и блокаторами ренин-ангиотензин-альдостероновой системы (РАAC) [5], хотя при их применении в комбинации с другими антигипертензивными препараами риск развития XСН не только не увеличивается, но может и уменьшаться $[5,13]$

В рекомендациях Европейского обшества кардиологов (European Society of Cardiology, ESC) по диагностике и лечению острой и хронической сердечной недостаточности (2016) [14] указывается, что контроль АД являетс важной составляюшей в комплексном ведении пашиента с ХСН, и преллагается использовать иАПФ (или БРА в случае непереносимости иАПФ), $\beta$-АБ, антагонисть минералокортикоидных рецепторов (АМР), диуретики, гидралазин и амлодипин [15] (или фелодипин [16]), для решения о нелевом уровне АД эти рекомендашии ссыл для рена европейские рекомендашии по АГ (2013) [17], преги- гая применять общепринятые целевые уровни АД при АГ и ХCH [14]. В рекомендациях Европейского общества по гипертонии (European Society of Hypertension, ESH) и Европейского общества кардиологов (European Society of Cardiology, ESC) по лечению АГ (2013) [17] целевые уровни АД были заявлены ниже $140 / 90$ мм рт. ст. для подавляющего большинства больных с АГ, в том числе при сочетании АГ и ХСН. Более того, в них высказываются сомнения в наличии дополнительных преимуществ от снижени систолического АД (САД) до уровня ниже 140 мм рт. ст. для лечения АГ у пациентов с ХСН (для СНсФВ и сердечной недостаточности с низкой фракцией выброса левого желудочка - СНнФВ), поскольку в ранее проведенных исследованиях имеет место недостаток надлежащих и единообразных результатов [17]

Российские клинические рекомендации по диагностике, профилактике и лечению $\mathrm{CH}$ (2018), разработанные экспертами Общества специалистов но сердечной недостаточности (ОССН), Российского кардиологического общества (РКО) и Российского национального обществ терапевтов (РНМОТ) [11], перечисляют лишь лекарственные средства, которые имеют хорошую доказательную базу для предотвращения развития XCH у пациентов с $\mathrm{A}$ $[5,10,13]$, и далее ссылаются на рекомендации по лечению АГ, принятые Американской коллегией кардиолого (American College of Cardiology - ACC) и Американской ассоциацией сердца (American Heart Association - AHA) в ноябре 2017 г. [10], в которых впервые указаны целевые значения снижения АД при лечении АГ у пациентов с ХCH как ниже 130/80 мм рт. ст. [10].

В новых рекомендациях ESC по диагностике и лечению АГ (ESH/ESC, 2018) [2] лекарственные препараты, ориентированные на лечение ХСН - иАПФ, БРА, $\beta$-АБ, АМР (спиронолактон, эплеронон), рекомендуются также и лля лечения АГ у пациентов с ХCH. Эксперты предлагают начинать антигипертензивную терапию у пациентов с ХCH (если она еще не начата) при АД>140/90 мм рт. ст. (для СНсФВ и СНнФВ). Однако до каких цифр необходимо снижать Ад при лечении АГ у пациентов с ХCH, остается неясным. ЭТо связано с тем, что в ряде исследований у пациентов с ХCH выявлен более высокий риск смерти и повторных госпитализаший при низких значениях АД (хотя интерпреташия данных затруднена из-за возможности обратной причинно-следственной связи), что говорит о шелесообразности избегать активного снижения АД (<120/70 мм рт. ст) [2]. В других исследованиях бө 
циентов могут быть достигнуты даже более низкие уровни АД, чем 120/70 мм рт. ст. из-за возможности профилактиче-
ского/зашитного эффекта лекарственных средств для леческого/защитн ХСН [14].

В сравнении с общей популяцией в западных странах отмечается высокая распространенность ХCH у лиц пожилого возраста (от 1 до $2 \%$ в общей популяции и до $10 \%$ улиц старше 70лет) [18]. В России более $65 \%$ больных ХСН имеют возраст 60 лет и старше, при этом за последние 18 ле средний возраст пацинас ХСН увеличился с 64 \pm 11 , 9 года

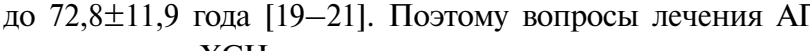
у страдающих ХСН пациентов пожилого и старческого возраста имеют особую актуальность. У пожилых пациентов чаще нарушены механизмы, поддерживающие гомеоста и жизненно важную перфузию органов, поэтому ожидае мые преимущества по сравнению с потенциальным вредом от лечения АГ будут зависеть от их функционального статуса и способности переносить лечение [2]. В рекомендациях ESH/ESC (2018) [2] целевые значения АД на фоне антигипертензивной терапии у пациентов 65 лет и старше составляют 130-139/70-79 мм рт. ст. при хорошей их переносимости, причем указана и нижняя граница безопасного снижения АД - не ниже 130/70 мм рт. ст., независимо от наличия сопутствующих заболеваний [2]. С другой сторонь, в ряде исследований показано, что у пациентов старческого возраста, даже без синдрома старческой астении, целево уровень АД может быть выше рекомендованного рабочей группой ESH/ESC (2018), причем более высокие значения целевого АД могут обусловливать уменьшение риска серьезных сердечно-сосудистых событий: инсульта, сердечной недостаточности (СН) исмертности ог всехпричин [8, 22-24]. Также существуют исследования, в которых обнаружено, что более низкие значения целевого АД могут быть сопряжены с наименьшей выживаемостью и увеличением смертности [25-28].

Таким образом, на сегодняшний день нет четкого предческого возраста, несмотря на то что лекарственные препараты для лечения АГ у пациентов с ХСН хорошо известнь. А в реальной клинической практике определение целевого уровня АД в большей степени зависит от осведомленности лечащего врача об этой проблеме. Данная статья представляет обзор исследований, в которых изучались целевые уровни снижения АД при лечении АГ у пациентов пожилого возраста с XCH.

\section{Целевые значения АД при лечении АГ у пациентов
с ХСН с СНнФВ}

Одним из ключевых показателей гемодинамики при СН является фракция выброса (ФВ) левого желудочка (ЛЖ). Она имеет большое прогностическое значение: чем меньше ФВ ЛЖ, тем хуже прогноз [11]. СНнФВ определяется при $\Phi B<40 \%$, что говорит о нарушении систолической функции ЛЖ [11]. У больных ХСН в анамнезе часто имеется АГ, однако после развития СНнФВ повышение АД может АГ, однако после развития СНнФВ повышение АД может иного рандомизировнного кинического исстегов
(РКИ), оценивающего целевой уровень АД (из большинс

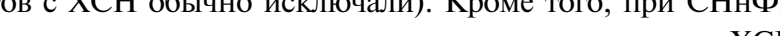
может развиться артериальная типотония, лечить ХСН у тал болых становится гораздо труднее, чем у больных

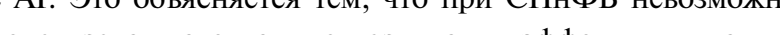
использовать адекватные дозы таких эффективных антивают отрицательное инотропное действие. Данные в пользу базнчения пациентам с СНнФВ иАНФ, БРА, $\beta$-АБ и АМР были получены в исследованиях, в которых их назначали для устранения избыточной стимуляции сердца симпатическими нервными стимулами и РААС, а не для снижения АД (в ряде этих исследований показатели АД даже не были указаны) [29]. Артериальная гипотония при СНнФВ являетс одной из причин отсутствия международных рекомендации по целевому уровню АД у больных с АГ и СНнФВ. Но в то же время существуют пациенты с повышенным уровнем АД 作 СНнФВ, что требует дополнительного назначения антигипертензивной терапии.

Хотя специально спланированных исследований, сравнивающих целевые значения АД при лечении АГ пациентов с СНнФВ не проводилось, имеется ряд исследований, которые показывают преимущества более высоких цифр АД у пациентов с СНнФВ по сравнению с боле низкими в плане спижения сердечно-сосудистых событи и смертности. Так, C. Raphael и соавт. [30] провели метаанализ 10 проспективных обсервационных исследований у пациентов с СНнФВ, в которых изучали возможную взаимосвязь АД и смертности от ХСН, и показали, что боле высокий уровень САД ассоциирован с лучшими исходами. В общей сложности были проанализированы данные 8088 Вациентов, с последующим наблюдением - 29222 человеко-лет. Пациенты, включенные в этот метаанализ, имели среднюю ФВ ЛЖ 28,3\%, среднее САД по всем включенным исследованиям составило 124,9 мм рт. ст. Показано, что более высокое САД было благоприятным прогностическим маркером при СНнФВ в отличие от населения в целом, цля которого оно является показателем худшего прогноза Снижение смертности в популяции пациентов со СНнФВ, связанное с повышением САД на 10 мм рт. ст., составило $13,0 \%$ (95\% доверительный интервал - ДИ - 10,6-15,4\%). Полученные результаты не зависели ни от этиологии ХСН, ни от факта применения иАПФ или $\beta$-АБ. Благоприятны эффект более высокого САД был значительнее выраже у пациентов с низким исходным уровнем САД, с $18 \%$ снижения смертности для пашиентов в самом низком тертило САД (среднее САД 109 мм рт. ст.) на каждые 10 мм рт. ст. повышения САД и $10 \%$ снижения смертности для пашиенто в самом высоком тертиле САД (среднее САД 145 мм рт. ст.) на каждые 10 мм рт. ст. повышения САД. Авторы предположили, что эта тенденция может сохраняться и каждое последуюшее увеличение САД на 10 мм рт. ст. (исходное САД от 160 до 170 мм рт. ст.), что, возможно, привело бы к гораз до меньшему снижению смертности, если таковое ворб имело бы место. Макси см в данно метанозе отмечено в исслеговани В. Нuуп и соавт. [31], в котором была выявлена лишь незначительная и статистически незначимая связь между САД и смертностью. Поэтому авторы предположили о существовани -образной взаимосвязи САД и смертности в популяци пациентов С СНнФВ. Следует обратить внимание на то

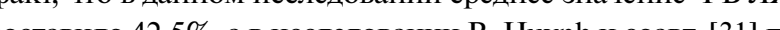
составило 42,5\%, а в исследовании В. Huynh и соавт. [31] по

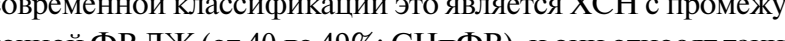
очной ФВ ЛЖ (ог 40до ческой дисфункцией [14].

В нелавно опубликованном исследовании S. Lee и соав 32] у пациентов с XCH обнаружена J-образная взаимосвяз АД и смертности от всех причин. В общей сложности в исследовании приняли участие 4487 пациентов, из которых 2581 имел СНнФВ (средняя ФВ ЛЖ 27,29 7,62\%, средни

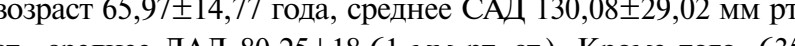
ст., среднее ДАД $80,25 \pm 18,61$ мм рт. ст.). Кроме того, 635 пациентов имели СНпФВ (средняя ФВ ЛЖ 44,68 $2,56 \%$ средний возраст 71 $\pm 12,94$ года, среднее САД 139,19 $\pm 31,09$ мм рт. ст., среднее ДАД $80,65 \pm 19,37$ мм рт. ст.), средний период наблюдения составил 2,2 года. У пациентов с СНнФ выявлена Ј-образная зависимость смертности от всех причин от уровня АД, с наименьшим риском при САД/ДАД 136,0/76,6 мм рт. ст. Причем смертность статистически значимо увеличивалась только при более низких уровнях АД и наблюдалась лишь тенденй к увеличению смертности при более высоком САД. Также было показано, что при более Низких значениях САД и ДАД у Пациентов с СНнФ увеличивается риск повторных госпитализаций по поводу ХCH (вторичная конечная точка).

В ретроспективном анализе многоцентрового рандомизированного плацебо-контролируемого клинического исследования эффективности дигоксина у пациентов с ХСDigitalis Investigation Group (DIG) [33, 34] M. Banach и соав [35] у 5747 пациентов с СНнФВ изучали связь САД 120 м рт. ст. (медиана САД $114 \pm 10$ мм рт. ст., средняя ФВ ЛЖ 29 $\pm 11 \%$, средний возраст $62 \pm 11$ лет) в сравнении с САД>12 мм рт. ст. (медиана САД $134 \pm 10$ мм рт.ст., средняя $Ф В$ ЛЖ $35 \pm 13 \%$, средний возраст $65 \pm 10$ лет) с отдаленными результатами. Первичной конечной точкой была смертность от всех причин в течение 5 лет, вторичная конечная точка включала смертность от конкретных причин и количество госпитализаций. В результате анализа полученных данных показано, что смерть от всех причин имела место у $35 \%$ пациентов с САД $\leq 120$ мм рт. ст. и $32 \%$ пациентов с САД>120 им рт. ст. в течение 5 лет наблюдения (относительньй риск - OP $-1,10 ; 95 \%$ доверительный интервал - ДИ $0,99-1,23 ; \mathrm{p}=0,088)$ в в том числе смертность от сердечно-сосудистых заболеваний (ОР 1,$15 ; 95 \%$ ДИ $1,01-1,30 ; \mathrm{p}=0,031)$ и смертность от СН (ОР 1,$30 ; 95 \%$ ДИ $1,08-1,57 ; \mathrm{p}=0,006)$ была выше у пашиентов с САД 120 мм рт. ст. Госпитализашия по поводу обострений сердечно-сосудистых заболева ний имела место у $53 \%$ пашиентов с САД 120 мх рт ст. и $49 \%$ пашиентов с САД>120 мм рт. ст. (ОР 1,$13 ; 95 \%$ ДИ 1,03-1,24; $\mathrm{p}=0,008)$, также в группе пашиентов с CAД $\leq 120$ мм рт. увеличивался риск госпитализашии от всех причин (ОР 1,10 ; $95 \%$ дИ $1,02-1,194 ; \mathrm{p}=0,017)$ и риск госпитализачии по воду обострений СН (OP 1,21; $95 \%$ ДИ 1,07-1,36; $\mathrm{p}=0,002$ ). Таким образом, в данном исследовании у пациенто СНнФВ исходный уровень САД 120 мм рт. ст. был ассоциирован с более высокой сердечно-сосудистой смертность. смертностью от $\mathrm{CH}$, а также с более высокой частото геспитализации в связи с обоспрением ХСН и других сер-

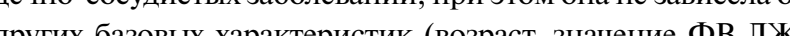
других базовых характеристик (возраст, значение ФВ ЛЖ (2)

В другом ретроспективном анализе этого же исследования DIG T. Lee и соавт. [36] у пациентов с СНнФВ изучали возможную ассоциацию между уровнями САД и ДАД, разделенных на несколько категорий, и общей смертностью. База данных включала 5747 пациентов (средний возрас $63 \pm 11$ лет, среднее значения ФВ ЛЖ 28 $\pm 9 \%$, более $80 \%$ всех пациентов принимали иАПФ и диуретики), медиана периода наблюдения составила 38 мес. Пациенты были классифицированы в соответствии с их базовым уровнем АД на групп по САД (<100, 100-109, 110-119, 120-129, 130-139, $140-149$ и $\geq 150$ мм рт. ст.) и на 5 групп по исходному уровнг ДАД ( $<60,60-69,70-79,80-89$ и $\geq 90$ мм рт. ст.). Результать показали, что в скорректированном анализе пропорциональных рисков Кокса уровень САД $<100$ мм рт. ст. ассоциировался с более высокой смертностью по сравнению с уровнем в контрольной группе (САД от 130 до 139 мм рт. ст.): ОР $1,80 ; 95 \%$ ДИ $1,36-2,38, \mathrm{p}<0,001)$. Пациенты с уровнем САД от 100 до 109 мм рл. с. имели второй по величине риск смерти (ОР 1,43; 95\% ДИ 1,14-1,80, p=0,002). Пациенты с уровнем САД от 120 до 129 мм рт. ст. также имели повышенный риск смерти по сравнению с контрольной группой (САД о 130 до 139 мм рт. ст.): ОР 1,$27 ; 95 \%$ ДИ $1,04-1,57, \mathrm{p}=0,021$. Группы с более высоким уровнем САД имели риск смерти, аналогичный риску в контрольной группе. В скорректированном анализе пропорциональных рисков Кокса уровен ДАД <60 мм рт. ст. был независимо связан с повышенно смертностью (ОР 1,64; 95\% ДИ 1,23-2,18, $\mathrm{p}=0,001$ ), так же как и уровень ДАД 60-69 мм рт. ст. (ОР 1,44; $95 \%$ ДИ 1,20$1,71, \mathrm{p}<0,001)$ в сравнении с контрольной группой (ДАД о 80 до 89 мм рт. ст.). Остальные группы по уровню ДАД имели риск смерти, аналогичный риску в контрольной группе. Статистически значимая тенденция более высокой смертности при более низких значениях АД $(\mathrm{p}<0,001)$ была очевидна после 1 года наблюдения и продолжала расти с увелинием периода наблюдения.

В субанализе базы данных EPRP (External Peer Review Program) национальной когорты ветеранов (Cohort of Veterans) и исследования DIG (Digitalis Investigation Grou САД и смертностью в зависимости от степени снижения ФВ ЛЖ. В результате было показано, что у пациентов от легкой до умеренно сниженной ФВ ЛЖ (30\% $Ф$ В ЛЖ<50\%) уровень САД имел нелинейную связь со смертностию: самая низкая смертность была в дипазоне САД от 130 до 140 мм рт. ст., при этом у пациентов с уровнем САД<110 мм рт. смертность статистически значимо увеличиватас. В базе данных EPRP статистически зночимо возрастала В базе данных EPRP статистически значимо возрастала смерт-
ность при уровне САД> 150 мм рт. ст., тогда как в базе дан- 
ных DIG была только тенденция к увеличению смертности при аналогичном уровне САД. У пациентов с сильно сниженной ФВ ЛЖ ( со смертностью, причем более высокий уровень САД бы ссоциирован с лучшими исходами

В исследовани BEST Thal (Beta Blocker Evaluation of Survival Trial) [38] у пациентов с прогрессирующей XCH с систолической дисфункцией было изучено влияние ис-

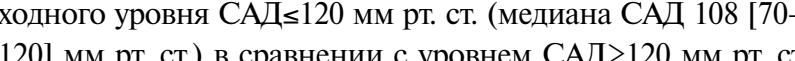
120] мм рт. ст.) в сравнении с уровнем САД>120 мм рт. ст. (медиана САД 134 [121-192] мм рт. ст.) на различные неблагоприятные исходы. В исследовании приняли участие 2708 пациентов, из которых отобрали для анализа 545 пар пациентов, сопоставимых по 65 базовым характеристикам (сред-

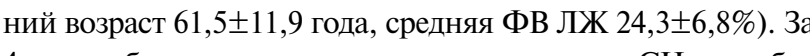
4 года наблюдения госпитализация по поводу СН потребовалась $38 \%$ пациентов с уровнем САД $\leq 120$ мм рт. ст. и $32 \%$ пациентов с уровнем САД>120 мм рт. ст. (ОР 1,33; $95 \%$ ДИ 1,04-1,69; $\mathrm{p}=0,023$ ). Смерть от всех причин имела место у $28 \%$ пациентов с уровнем САД $\leq 120$ и $30 \%$ пациентов с уровнем САД>120 мм рт. ст. (ОР 1,13; $95 \%$ ДИ 0,86-1,49, $\mathrm{p}=0,369)$. Данное исследование показало, что у пациентов c прогрессирующей ХCH с систолической дисфункциеи исходный уровень САД $\leq 120$ мм рт. ст. ассоциирован с повышенным риском госпитализации по поводу $\mathrm{CH}$, но не со смертностью от всех причин.

Субанализ итальянского реестра пациентов с застойной CH IN-CHF (Italian Network on Congestive Heart Failure) реестра (IN-CHF) [39] у пациентов пожилого возраста на основании анализа данных 3327 амбулаторных больных выявил независимые предикторы смертности в течение первого года наблюдения, одним из которых явился более низкий уровень САД ( $<100$ мм рт. ст.). Данный анализ включа 2294 пациентов в возрасте до 70 лет (средний возраст - $58 \pm 9$ лет, 77,4\% пациентов с ФВ ЛЖ<40\%) и 1033 пациента 70 лет и старше (средний возраст $75 \pm 5$ лет, 67,4\% пациентов с ФВ лж<40\%).

в субанализе исследования CHARM (Candesartan in Heart failure: Assessment of Reduction in Mortality and morbidity) [40] основные результаты заключались в том, что у пациентов с СНнФВ $(<40 \%)$ низкий уровень САД, так же как и низкий уровень ДАД, были ассоциированы с худшими клиническими исходами. В этом исследовании 4576 пациентов с XCH II-IV класса по NYHA (New York Heart Association Functional Classification) и ФВ ЛЖ $<40 \%$ были рандомизированы на две группы: пашиенты, принимаюшие кандесартан (средний возраст $65,1 \pm 10,9$ года, средняя ФВ ЛЖ $29 \pm 0,8 \%$, и пашиенты, принимаюшие плашебо

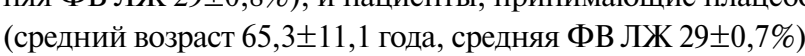
Первичной конечной точкой была сердечно-сосудистая смертность или госпитализация по поводу обострения ХСН и смерть от всех причин. Результаты исследования СНАRM P. Meredith и соавт. [41] проанализировали в соответствии с разными категориями исходного уровня САД и ДАД- 6 категорий для исходного уровня САД ( $\leq 100,101-110,111-120$ 121-130, 131-140 и $\geq 141$ мм рт. ст) и 4 категории для исходного уровня ДАД ( $\leq 60,61-70,71-80$ и $\geq-81$ дл рт. ст). В среднем через 40 мес. наблюдения было показано, что пациенты с более низким уровнем САД (или ДАД) в начал иследования имели больший риск смерти от сердечно-сосеи чем пациен с более высоким уровнем АД, причем это

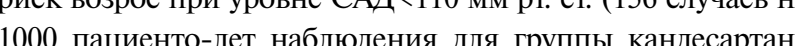
1000 пациенто-лет наблюдения для группы кандесартан и 197 случаев на 1000 пациенто-лет наблюдения для груп1000 пцео) и особенно ниже 100 мм рт. ст. (228 случаев на

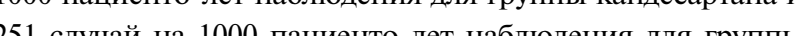
251 слуай на 1000 пациенто-лет наблюдения для трупін .

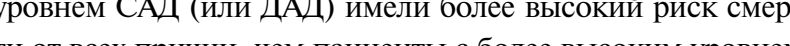
АД: для САД<100 мм рт. ст. (154 случая на 1000 мовне

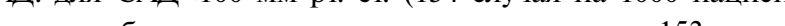

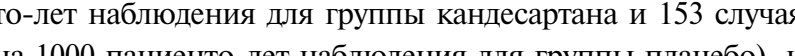
для САД<110 мм рт. ст. (108 случаев на 1000 пациенто-лет наблюдения для группы кандесартана и 127 случаев на 1000 иенто-лет наблюдения для группы плацебо).

В субанализе исследования PARADIGM-HF (Prospective Comparison of angiotensin receptor-neprilysi nhibitor (ARNI) with an angiotensin-converting enzyme (ACE) inhibitor to Determine Impact on Global Mortality and Morbidity in Heart Failure trial ) [42] - B. Michael и соавт. [43] анализировали данные 8399 пациентов, включенных В эло исследование, с XCH II-IV класса по NYHА и ФВ ЛЖ $<40 \%$, щие LCZ696 (средний возраст 63,8 $\pm 11,5$ года, средняя ФВ

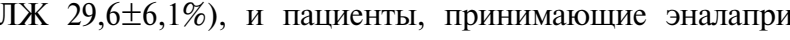

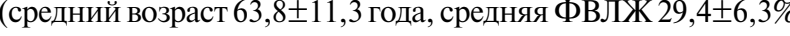
в дополншие к рекомендуемой терапии. Авторы оценили риск развития первичной конечной точки (совокупность смерти от сердечно-сосудистых причин или госпитализация по поводу СН) и вторичных исходов (смерть от всех причин и общий клиниеский балл опросника по кардиомиопатии KCCQ - Kansas City Cardiomyopathy Questionnaire) B cooTветствии с разными категориями исходного уровня САД. Для этого пациентов разделили на несколько подгрупп (исходное САД $<110$, от 110 до $<120$, от 120 до $<130$, от 130 до $<140$ и $\geq 140$ мм рт. ст.). Медиана периода наблюдения составила 27 мес., после чего было показано, что риск госпитализации по поводу СН был выше у пациентов с более низким уровнем САД (САД<110 мм рт. ст.) и с уровнем САД>140 мм рт. ст., то есть имелась Ј-образная связь между САД и госпитализацией по поводу СН. Риск сердечно-сосудистой смерти увеличивался у пациентов с уровнем САД $<110$ мм рт. ст. а примерно с уровня САД $\geq 120$ мм рт. ст. риск сердечно-сосулистой смерти сохранялся на одном и том же уровне.

Целью ретроспективного продольного исследования F. Schmid и соавт. [44] было определить влияние из менений уровня АД на прогноз и смертность в когорте пациентов с ХСН. Всего в исследование были включень 927 пашиентов, медиана возраста (на момент начала исследования) 58 (49-67) лет, медиана ФВ ЛЖ 34 (25-47) $\%$, число пациентов с ФВ ЛЖ $<50 \%$ составило $743(80,2 \%)$. Среднй уров САД в начале исслегования состар лрял 117 (104-130) мм рт. ст., средний уровень ДАД - 76 (68-82) ми рт. ст. В течение периода набюоген 290 пани- ентов достигли первичной конечной точки (220 пациентов умерли, 70 пациентов перенесли трансплантацию сердца). Медиана выжиасмоси составила 7,7 $(6,6-9,8)$ года. Лучшая выживаемость отмечена у пациентов с наиболее стабильными значениями АД в течение периода наблюдения. Изме лее чем на \pm 10 мм рт. ст. в год приводили к статистически значимо худшему результату (ОР 1,8 и 2,0, соответственно, p $<0,001$ для обоих случаев). При сравнении влияния САД на выживаемость показано, что пациенты с самыми высо-
ким уровнем САД ( 2130 мм рт. ст.) имели лучший прогноз, ким уровнем САД ( 2130 мм рт. ст.) имели лучший прогноз, а пациенты с самым низким САД (<90 мм рт. ст.), напро7ив, имели самую высокую смертность (ОР 12,1 ,

Целевые значения АД при лечении АГ у пациентов с ХСН с сохраненной ФВ ЛЖ (СНсФВ)

Пациенты с СНсФВ определяются как имеющи ФВ $>50 \%$ и не имевшие иной патологии со стороны сердц (например, клапанных нарушений или заболеваний перикарда) [11]. При подозрении на СНсФВ эхокардиографическое исследование должно подтвердить наличие структурных и/или функциональных нарушений работы сердца. К структурным изменениям относят увеличение левого предсердия (индексированный объем левого предсердия $>34$ мл/м²) и ГЛЖ (индекс массы миокарда ЛЖ $>115$ у мужчин и $>95 \mathrm{r} / \mathrm{M}^{2}$ у женщин) [11]. АГ более типична для больных СНсФВ, однако в исследованиях с конечными точками, в которые Специально включали именно таких больных, неконтролируемая АГ встречалась редко, так как эти пациенты получали массивную фоновую терапию, включавшую антигипертензивные препараты.

тана при СНсФВ (Irbesartan in Heart Failure with Preserved Ejection Fraction Study, [-PRESERVE) [45] приняли участие 4128 пашиентов старше 60 лет (средний возраст $72 \pm 7$ лет), имеющих XCH II-IV стадии (по NYHA) с ФВ ЛЖ не менее 45\%. Случайным образом пациенты были рандомизированы в группу приема ирбесартана (представитель класса БРА) 300 мг/сут. или в группу плацебо. Среднее значение ФВ лЖ для группь ирбесартана составляло $59 \pm 0,9 \%$, для группы плацебо $60 \pm 0,9 \%$. В качестве сопутствующей терапии все пациенты принимали другие антигипертензивные препараты. В ходе исследования доля пациентов, получавших иАПФ, выросла с $25 \%$ в обеих группах в начале исследования до $39 \%$ в группе ирбесартана и $40 \%$ в группе плацебо, использование спиронолактона возросло с $15 \%$ в обеих группах в начале исследования до $28 \%$ в группе ирбесартана и $29 \%$ в группе плацебо, а использование $\beta$-АБ возросло с $59 \%$ в группе ирбесартана и $58 \%$ в группе плацебо до $73 \%$ в обеих группах. На момент начала исследования средний уровень САД в группе ибесартана составил 137+15 мм рт. ст. в группе плацебо - $136 \pm 15$ мм рт. ст., ДАД $-79 \pm 9$ мм рт. ст в обеих группах. Между исходным уровнем и 6 мес. уровень САД снизился в среднем на $3,8 \pm 18,0$ мм рт. ст., ДАД - на $2,1 \pm 10,5$ мм рт. ст. в группе ирбесяртана и в группе плагебо на $0,2 \pm 17,6$ мм рт. ст. и $0,2 \pm 10,4$ мм рт. ст. соответственно. Эти значения в обеих группах сохранялись на протяжении все исследования. В результате в среднем за 49,5 мес. наблюдения различий между группой ирбесартана и группо пебо по первичной (комбинированная точка включала в себя смерть от любой причины или госпитализацию по поводу ухудшения ХCH, инфаркта миокарда, инсульта, нестабильной стенокардии, желудочковой или предсердной аритмии или инфаркт миокарда или инсульт, возникший во время любой госпитализашии) и вторичны компоненты первичного исхода: смерть от любой причины и госпитализашия по сердечно-сосудистым причинам, комбинированный исход ХСН - смерть в результате обострения ХCH или внезапная смерть или госпитализация в результате обострения XCH - изменение общего балла качества жй для пациентов с XСH по шкале MLHFQ (Minnesota Living with Heart Failure scale) за 6 мес., изме нение уровня NT-proBNP ( $\mathrm{N}$-terminal pro brain natriuretic peptide, где $\mathrm{N}$ - терминальный участок мозгового натрийуретического пептида) в плазме крови через 6 мес., смерть от сердечно-сосудистых причин (нефатальный инфарк миокарда/нефатальный инсульт, смерть от сердечно-сосудистых причин) конечным точкам обнаружено не было. Таким образом, в данном исследовании не выявлено дополнительных преимуществ от снижения уровня АД для пациентов СНсФВ

Из 4487 пациентов описанного ранее исследования S. Lee и соавт. [32] 1130 пациентов имели СНсФВ (средняя

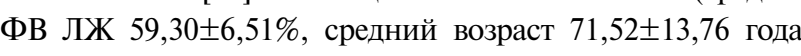
средний уровень САД $136,65 \pm 30,16$ мм рт. ст. и средний уровень ДАД 77,76 18,21 мм рт. ст.). Наблюдали Ј-образную ассоциацию между смертностью от всех причин и исходным уровнем АД, с наименьшим риском при уровне САД/ДАД 127,9/72,7 мм рт. ст. Для риска повторных госпитализаций по поводу обострения ХСН (вторичная конечная точка) также наблюдали Ј-образную ассоциацию с исходным уровнем САД, с наименьшим риском при уровне САД 127,6 мм рт. ст. ( $\mathrm{p}=0,0068)$. Кроме того, риск повторных госпитализаций увеличивался при более низких значениях ДАД.

В недавнем наблюдательном исследовании Treatment in Hospitalized Patients with Heart Failure) [46 проведен анализ данных 1802 пациентов (средний возрас

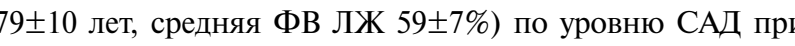
выписке, при этом сравнивали группу пациентов с уровнем САД<120 мм рт. ст. (средний уровень САД при поступлении $114 \pm 12$ мм рт. ст., средний уровень САД на момен выписки $109 \pm 8$ мм рт. ст.) и группу больных с уровнем САД 2120 мм рт. ст. (средний уровень САД при поступлении $137 \pm 16$ мм рт. ст., средний уровень САД на момент выписки $135 \pm 13$ мм рт. ст.). В качестве первичной конечной точки рассматривали смертность от всех причин и повторную госпитализацию в течение 30 дней, 1 года и всего период наблюдения (период наблюдения достигал 6 лет, мелиан периода наблюдения - 2,1). Вторичные исходы рилючали повторную госпитализашию по всем причинам и 2 комбинированные конечные точки: повторная госпитамизаи по всем причинам и смертности по всем причинам или повторная госпитализашия в связи с декомпенсашией СН исмертности по всем причинам. В результате в течени 30 
дней смерть от всех причин имела место у $10 \%$ пациентов с уровнем САД<120 мм рт. ст. и 5\% пациентов с уровнем Уровень САД $<120$ мм рт. ст. также был связан с более высоким риском смертности через 1 год (39\% против $31 \%$; соким риском смериоси через 1 тод (Ј9\% против З1\%; периода наблюдения 2,1 года (OP 1,17; $95 \%$ ДИ 1,05-1,30; $\mathrm{p}=0,005)$. Уровень САД<120 мм рт. ст. был ассоциирован с более высоким риском повторной госпитализашии по поводу СН в течение 30 дней (ОР 1,47; 95\% ДИ 1,08-2,01; $p=0,02$ ), однако через год или через 6 лет наблюдения такой взаимосвязи выявлено не было. ОР развития комбинированной конечной точки, повторной госпитализации по поводу СН или смертности от всех причин, связанный с уровнем САД $<120$ мм рт. ст., через 30 дней составил 1,71 (95\% ДИ 1,34-2,18; p<0,001), через 1 год - 1,21 (95\% ДИ $1,07-1,38 ; \mathrm{p}=0,004)$ и в целом за медиану периода на-
блюдения $-1,12$ (95\% ди $1,01-1,24 ; \mathrm{p}=0,03)$. Результаты исследования ОРTIMIZE-HF показали, что для пациентов с СНСФВ уровень САД 120 мм рт. ст. в значительно степени связан с худшими исходами (увеличение риска смертности и повторных госпитализаций).

ЛИTEPATYPA/REFERENCES

Rapsomanikik E., Timmis A, George J., Pujades-Rodriguez M., Shah A.D.
Denaxas S., White I.R., Caulfield M.J., Deanfield J.E., Smeeth L., Williams B., Hingorani A., Hemingway H. Blood pressure and incidence of twelve

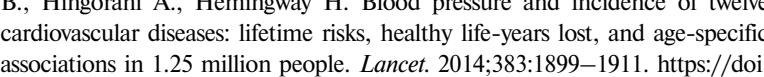
associations in 1.25 million peopl.
$10.1016 / 50140-6736(14) 60685-1$.

2018 ESC/ESH Guidelines for the management of arterial hypertension. $E w$ Effects Morbidity of Treatment on in Hypertension: II. Results in Pat With Diastolic Blood Pressure Averaging 90 Through $114 \mathrm{~mm}$ Hg. JAMA
$1970 \cdot 213(7) \cdot 1143$. htps://doi: $10.1001 /$ jama 1970.0317033025003 1970:213(7):11433. https:///doi:10.10001 jama.1970.03170330025003. persons with isolated systolic hypertension. SHEP Cooperative Research Group.
JAMA: The Journal of the American Medical Association. 1997; 278(3):212-6. https://doi:10.1001 Joul jama. 278.3.2.212

Thomopoulos C., Parati G., Zanchetti A. Effects of blood pressure lowering on outcome incidence in hypertension: 7. Effects of more vs. less intensive overview and meta-analyses of randomized trials. Journal of Hypertension.
ond BakerDW.Prever 46. https:///doi:10.1054/ jeaf. 2002.0000333.
SPRINT Research Group, Wright J.T. J., Williamson J.D., Whelton P.K. SPRINT Research Group, Wright J.T. Jr., Williamson J.D., Whelton P.K.
Snyder J.K., Sink K.M. Rocco M.V., Reboussin D.M., Rahman M., Oparil S. Cushman W.C., Cheung A.K. Ambrosius W.T. A randomized trial of intensive
versus standard blood-presure control. $N$ Engl I Med. 2015;3733:2103-2116 https://doi:10.1056/NEJMoa151193.

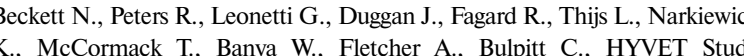
Group. Subgroup and per-protocol analyses from the Hypertension in the Very Elderly Trial. J Hypertens. 2014;32:1478-1487. https://doi:10.1097/

ALLHAT Officers and Coordinators for the ALLHAT Collaborative Research angiotensin-converting enzyme inhibitor or calcium channel blocker vs diuretic Trial (ALLHAT). JAMA. 2002;288:2981-2997.

10. Whelton P..., Carey R.M., Aronow W.S., Casey Jr. D.E., Collins K.J., Dennison
Himmelfarb C., DePalma S.M., Gidding S., Jamerson K.A., Jones D.W. Himmelfarb C., DePalma S.M., Gidding S., Jamerson K.A., Jones D.W.
MacLaughlin E.J., Muntner P., Ovbiagele B., Smith J. S.C., Spencer C.C. Stafford R.S., Taler S.J., Thomas R.J., Williams Sr. K.A., Williamson J.D.
Wright J.J.J., 2017 ACC / AHA /AAPA/ABC/ACPM /AGS / APhA / ASH
Таким образом, рекомендации ведущих кардиологичесих сообшеств по целевым цифрам АД у пациентов с ХСН АГ эксраполированы из исследований, проводившихс у групп населения без ХСН, поскольку прямые данные паииентов с ХСН ограничены. Однако результаты многих исследований по изучению взаимосвязи уровня АД и сердечно-сосудистых событий и смертности показывают, что боле низКий уровень САД (<120 мм рт. ст.) и ДАД ( $<80$ мм рт. ст.) у пациентов с XСН может бынь ассоциирован с развитием неблагоприяных сердечно-сосудистых событии, особенно Упациентов С СннФ. Проииворечивы данные относитель. 4иентов с ХCH, потому как одни исследования показываю примущества в снижении сердечно-сосудистых событи при более высоких значениях АД, тогда как другие показьвают противоположные результаты. В свете вышеизложенного необходимо проведение специально спланированны проспекиивых рандомизированных клинических исследо作 ов с ХСН, в том числе с учетом ФВ ЛЖ.

\section{Авторы заявляют об отсутствии конфликта интересов.}

/ASPC/NMA / PCNA Guideline for the Prevention, Detection, Evaluation, and Management of High Blood Pressure in Adults. Jourzal of the America.
College of Cardiology, 2018; 71 (19): $12127-248$. doi:10.1016/j.jacc.2017.11.006.

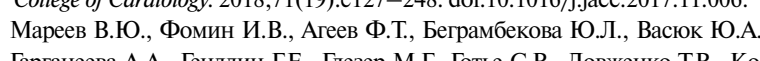

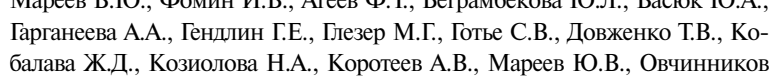
балава Ж.Д., Козиолова Н.А., Коротеев А.В., Мареев Ю.В., ОВчинников

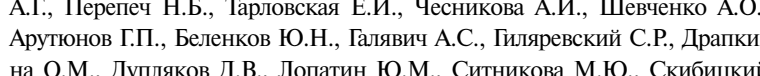
на О.М., Дупляков Д.В., Лопатин Ю.М., Ситникова М.Ю., Скибицкии
В.В., Шляхто Е.В. Клинические рекомендации ОССН - РКО - РНМО Сердечная недостаточность: хроническая (ХCH) и острая декомпенси-

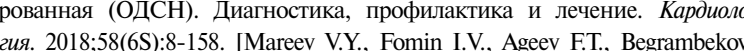
Y.L., Vasyuk Y.A., Garganeeva A.A., Gendlin G.E., Glezer M.G., Gautier S.V. Dovzhenko T.V., Kobalava Z.D., Koziolova N.A., Koroteev A.V., Mareev Y.V.,

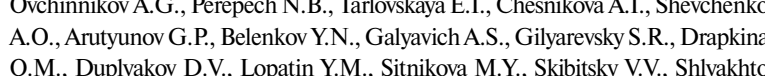
E.V. Russian Heart Failure Society, Russian Society of Cardiology. Russian Scientific Medical Society of Internal Medicine Guidelines for Heart failure

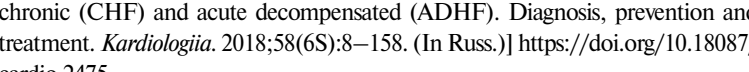
cardio. 2475 .

Thomopoulos C., Parati G., Zanchetti A. Effects of blood pressure-lowering
treatment 6 6 Prevention of hent fialure and treatment. 6. Prevention of heart failure and new-onset heart failure
meta-analyses of randomized trials. $J$ Hypertens. 2016;33:373-384. https: $/ /$. doi:10.1097/HJH. 000000000000000848.

Julius S., Kjeldsen S.E., Weber M., Brunner H.R., Ekman S., Hansson L.
Hua T., Laragh J., McInnes G.T., Mitchell L., Plat F., Schork A. Smith B, Zanchetti A.: VALUE trial group. Outcomes in hypertensive patients at hish cardiovascular risk treated with regimens based on valsartan or anlodipine the VALUE randomised trial. The Lancet. 2004;363(9426):2022-31. https://
doi:10.1016/S0140-6736(04) 64651 -9

Ponikowski P., Voors A.A., Anker S.D., Bueno H., Cleland J.G.F., Coats
A.J.S., Falk V., Gonzalez-Juanatey J.R., Harjola V.P., Jankowska E.A., Jessup M., Linde C., Nihoyannopoulos P., Parissis J.T., Pieske B., Riley J.P., Rosan G.M.C., Rullope L.M., Ruschitizka F., Rutten F.H., van der Meer P.; ESC
Scientific Document Group. $2016 \mathrm{ESC}$ Guidelines for the dianosis treatment of acute and chronic heart failure: The Task Force for the diagnosis and treatment of acute and chronic heart failure of the European Society of
Cardiology (ESC) Developed with the special contribution of the Heart Failure Cardiology (ESC) Developed with the special contribution of the Heart Failure
Association (HFA) of the ESC. Eur Heart J. 2016;37 (27):2129-2200. https:// doi:10.1093/eurhearti/hhw128.
15. Packer M., O'Connor C.M., Ghali J.K., Pressler M.L., Carson P.E., Belkin
R.N., Miller A.B., Neuberg G.W. Frid D., Wertheimer J.H.C Cropp A.B. R.N., Miller A.B., Neuberg G.W., Frid D., Wertheimer J.H., Cropp A.B
DeMets D.L. Effect of amlodipine on morbidity and mortality in severer chronic heart failure. Prospective Randomized Amlodipine Survival Evaluation
Study Group. $N$ Engl J Med. 1996;335(15):107-14. https://doi:10.1056/

16. Cohn J.N., Ziesche S., Smith R., Anand I., Dunkman W.B., Loeb H., Cintron

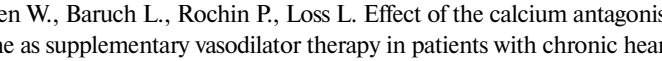
failure treated with enalapril: V-HeFT III. Vasodilator-Heart Failure Tria

Mancia G., Fagard R., Narkiewioz K. Redon J.

Christiaens T., Cikikova R., De Backer G., Dominiczak A., Galderisi M.,

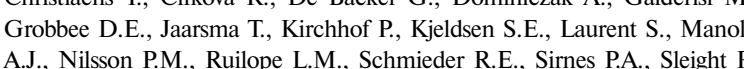
Viigimaa M., Waeber B., Zannad F., Redon J., Dominic Nilson P.M., Burnier M., Viigimaa M., Ambrosioni E., Caufield M., Coca A., Olsen M.H., Schmieder R.E., Tsiouffis C., van de Borne P., Zamorano J.L. C.chenbach S., Baumgartner H., Bax J.J., Bueno H., Dean V., Deaton C., Erol P., Sirnes P.A., Tamargo J.L., Tendera M., Torbicki A., Wjins W., Windecker S Clement D.L., Coca A., Gillebert T.C., Tendera M., Rosei E.A., Ambrosio Gest S., Derumeaux G.A., Erdine S., Farsang C., Funck-Brentano C.,
Gerc V., Germano G., Gielen S., Haller H., Hoes A.W., Jordan J., Kahan T., Gerc V., Germano G., Gielen S., Haller H., Hoes A.W., Jordan J., Kahan T, Komajda M., Lovic D., Mahrholdt H., Olsen M.H., Ostergren J., Parati G
Perk J, Polonia I. Popescu BA. Reiner Z Ryden L Sirenko Y. Stanto A., Struijker-Boudier H., Tsioufisis., van de Borne P., Vlachopoullos C., Volpe M., Wood D.A. $2013 \mathrm{ESH} / \mathrm{ESC}$ guidelines for the management of arteria hypertension: the Tasks Force for the Management of Atterial Hypertension of the European Society of Hypertension (ESH) and of the European Society or
Cardiology (ESC). Eur Heart J. 2013;34:2159-2219. https://doi: 10.1097/01 Card.00000431740.32696.cc.

18. Mosterd A., Hoes A.W. Clinical epidemiology of heart failure. Heart. 2007;93

(9): $1137-46$. https://doi:10.1136/hrt.2003.025?

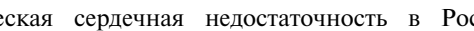
сийской Федерации: что сегодня мы знаем и что должны де-
лать. Российский кардиологический ж⿻рнал. 2016;(8):7-13. [Fоті
I. C.

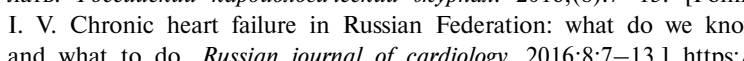
doi:10.15829/1560407120168713.

20. Фомин И.В., Беленков Ю.Н., Мареев В.Ю., Агеев Ф.Т., Бадин Ю.В Галяввч А.С., Даниелян М.О., Камалов Г.М., Колбин А.А., Кечеджие

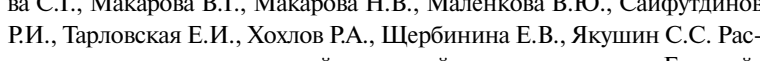
пространенность хронической сердечной недостаточности в Европей-
ской части Российской Фелерации - данные ЭПОХА - ХСН (часть

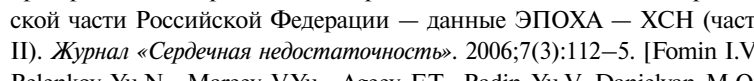
Kamalov G.M., Kolbin A.A., Kechedzhieva S.G., Makarova V.G.,., Makarova N.V., Malenkova V.Yu., Sayfutdinov R.I, Tarlosskaya E E.I., Khokhlov R.A. Shcherbinina E.V., Yakushin S.S. Prevalence of chronic heart failure in
European part of Russian Federation - Data of AGECHF (Part II). Russian Heart Failure Journal. 2006; 7 (1):112-5. (In Russ.)

21. Фомин И.В., Бадин Ю.В., Поляков Д.С., Беленков Ю.Н., Марее В.Ю., Агев Ф.Т. Артемвьва Е.Г., Галявич А.С., Камалов Г.М., КечедСайфутдинов Р.И., Смирнова Е.А., Тарловская Е.И., Щербинина Е.В. Якушин С.С. Предгипертония: как часто встречается данное состоя-
ние сертечн-осупистой системы у граждан Европейской части Росние сердечно-сосудистой системы у граждан Европейской части Рос
сии (даннье исследования ЭПОХА-АГ, 2002-2007 гг.). Современные

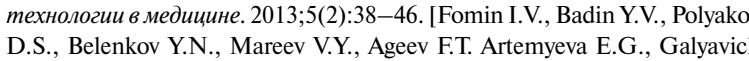
A.S., Kamalov G.M., Kechedzhieva S.G., Koziolova N.A., Malenkou V.Yu., Podzemelenikov E.V., Sayfutdinov R.I., Smirnova E.A.,., Tarlovskaya
E.I., Shcherbinina E.V., Yakushin S.S. Prehypertension: how often this cardiovascular condition occurs in citizens of European Russia (EPOCH-
AH, 2002-2007). Sovremennve tehnologii v medicine. 2013:5(2):38-46 (In AH, 2002-2007). Sovremennye tehnologii v medicine. 2013;5(2):38-46 (In
Russ ). 22. Ruans.).

van Bemmel T., Gussekloo J., Westendorp R.G., Blauw G.J. In a populationbased prospective study, no association between high $J$ lood pressure and
mortality after age 85 years. $J$ Hypertens. 2006;24(2):287-92. hitps: $/ /$ doi:10.1097/01.hih..0000200513.48441.8e.
23. Ravindrarajah R., Hazra N.C., Hamada S., Chartton J., Jackson S.H.D.
Dregan A., Gullifiord M.C. Systolic blood pressure trajectory, frailty and all-cause mortality $>80$ years of age: cohort study using electronic
health records. Circulation. 2017;135(24): 2357-68. https://doi:10.1161/ health records. Circulation. 2017;135(24): 2357-68. https://doi:10.116/
CIRCULATIONAHA.116.026687. Blom J.W., de Ruijter W., Witteman J.C., Assendelft W.J., Breteler M.M. Hofman A., Gussekloo J. Changing prediction of mortality by systolic
blood pressure with increasing age: the Rotterdam study. Age (Dordr)
2013:35(2):431-8 https://doi:10.1007//11357-011-9349-7. Poortviet R.K., Blom J.W., de Craen A.J,, Mooijaart S.P., Westendorp R.G. Assendelft W.J., Gussekloo J., de Rujiter W. Low blood pressure predicts
increased mortality in very old age even without heart failure: the Leiden $85-$ increased mortality in very old age even withou
plus study. Eur $J$ Heart Fail. $2013 ; 15(5): 528-33$.

Streit S., Poortvliet RKE, Gussekloo J. Lower blood presure antihypertensive treatment is associated with higher all-cause mortality an accelerated cognitive decline in the oldest-old-data from the Leiden 85-plu
study. Age Ageing. 2018; 8 : $1-6$. https://doi:10.1093/ageing/afy072 study. Age Ageing. 2018;(8): 1-6. hthps:///doi:10.1093//ageing/affyo72.
Molander L., Lovheim H., Norman T., Nordstrom P., Gustafson Y. Lower systolic blood pressure is associated with greater mortality in people aged 8.5
and older. $J A m$ Geriatr Soc. 2008;56(10):1853-9. https://doi:10.1111/.1532-

28. Mattila K., Haavisto M., Rajala S., Heikinheimo R. Blood pressure and five year survival in the very old. Br Med J (Clin Res Ed). 1988;296(6626):887-9.
Rutten F.H., Zuithoff N.P., Halk F., Grobbee D.E., Hoes A.W. Beta-Blocker may reduce mortality and risk of exacerbations in patients with chron

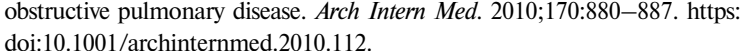

Raphael C.E., Whinnett Z.I., Davies.J.E., Fontana M.,. Ferenczi E.A., Manisty
C.H., Mavet J., Francis D.P. Quantifining the paradoxical effect of highe systolic blood pressure on mortality in chronic heart failure. Heart. 2009; systolic blood pressure on mortality in chronic hean
95:56-62. htpp:///doi:10.1136//rt.2007.134973. Huynh B.C., Rovner A., Rich M.W. Long-term survival in elderly patients hospitalized for heart failure: 14-year follow-up from a prospective
randomized trial. Arch Intern Med. 2006; $166: 1892-8$. https://doi:10.1001/ Lee S.E., Lee H.Y., Cho H.J., Choe W.S., Kim H., Choi J.O., Jeon E.S.
Kim M.S., Hwang K.K., Chae S.C., Baek S.H., Kang S.M., Choi D.J., Yoo Kim M.S., Hwang K.K., Chae S.C., Baek S.H., Kang S.M., Choi D.J., Yoo
B.S., Kim K.H., Cho M.C., Kim J.J, Oh B.H. Reverse J-Curve Relationship B.S., Kim K.H., Cho M.C., Kim J.J., Oh B.H. Reverse J-Curve Relationship
Between On-Treatment Blood Pressure and Mortality in Patients With
Heart Eailure. JICC Heart Fail. 2017; 5 (11):810-819. https://doi:10.1016/. jchf.2017.08.015.

Rationale, design, implementation, and baseline characteristics of patients in the DIG trail: alarge, simple, long-term trial to evaluate the effect of digitalis on
mortality in heart failure. Control Clin Trials. 1996;17:77-97.

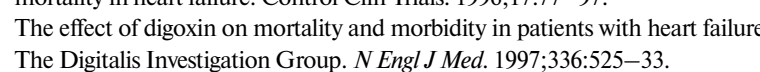
The Digitalis Investigation Group. N Engl J Med. 1997; $336: 525-33$ -
Banach M., Bhatia V., Feller M.A., Mujib M., Desai R.V., Ahmed M.I. Guichard J.L., Aban I., Love T.E., Aronow W.S., White M., Deedwaniz long-term outcomes in ambulatory patients with chronic mild to moderate
heart failure. $A m J$ Cardiol. 2011;107(8):1208-14. https://doi:10.1016/j

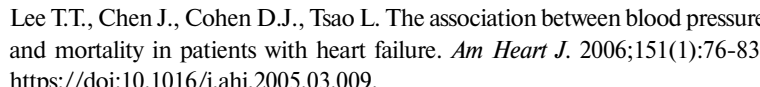
https.//doi:10.1016/.ahj.2005.03.009.
Ather S., Chan W., Chillar A., Aguilar D., Pritchett A.M., Ramasubbu K. Wehrens X.H., Deswal A., Bozkurt B. Association of systololic blood pressure with mortality in patients with heart failure with reduced ejection fraction: a
complex relationship. Am Heart J. 2011;161(3):567-73. https://doi:10.1016/3 complex relatio
ahj.2010.12.009.

Desai R.V., Banach M., Ahmed M.I., Mujib M., Aban I., Love T.E., White
M., Fonarow G., Deedwania P., Aronow W.S., Ahmed A. Impact of Baseline Systolic Blood Pressure and Long-Term Outcomes in Patients with Advanced

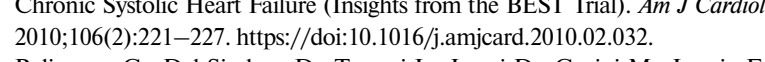

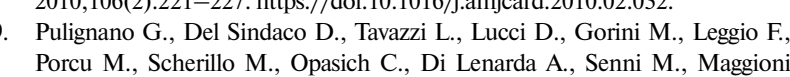
Porcu M., Scherillo M., Opasich C., Di Lenarda A., Senni M., Maggion
A.P.; IN-CHF Investigators. Clinical features and outcomes of elderly outpatients with heart failure followed up in hospital cardiology units: data
from a large nationwide cardiology database (IN-CHF Registry). Am Heart $J$.

2002; $143(1): 45-55$.
Young J.B., Dunlap M.E., Pfeffer M.A., et al,., for the Candesartan in Heart Falure Assessment or mediction. in Mortality and Morbidity (CHARM
Investigators and Committees. Mortality and morbidity reduction with candesartan in patients with chronic heart failure and left ventricular systolic dysfunction: results of the CHARM low-left ventricular ejection fraction trials. Circulatis. 
41. Meredith P.A., Ostergren J., Anand I., Puu M., Solomon S.D., Michelson E.L., Olofsson B., Granger C.B., Yusuf S., Swedberg K., Pfeffer M.A., McMurray J.J. Clinical outcomes according to baseline blood pressure in patients with a low ejection fraction in the CHARM (Candesartan in Heart Failure: Assessment of Reduction in Mortality and Morbidity) Program. J Am Coll Cardiol. 2008;52(24):2000-7. https://doi: 10.1016/j.jacc.2008.09.011.

42. McMurray J.J., Packer M., Desai A.S., Gong J., Lefkowitz M.P., Rizkala A.R., Rouleau J.L., Shi V.C., Solomon S.D., Swedberg K., Zile M.R. PARADIGMHF Investigators and Committees. Angiotensin-Neprilysin Inhibition versus Enalapril in Heart Failure. N Engl J Med. 2014;371(11):993-1004. https:// doi:10.1056/NEJMoa1409077.

43. Boehm M., Young R., Jhund P.S., Solomon S.D., Gong J., Lefkowitz M.P., Rizkala A.R., Rouleau J.L., Shi V.C., Swedberg K., Zile M.R., Packer M., McMurray J.J.V. Systolic blood pressure, cardiovascular outcomes and efficacy and safety of sacubitril/valsartan (LCZ696) in patients with chronic heart failure and reduced ejection fraction: results from PARADIGM-HF. Eur Heart J. 2017;38(15):1132-1143. https://doi:10.1093/eurheartj/ehw570.
44. Schmid F.A., Schlager O., Keller P., Seifert B., Huang R., Froehlich G.M., Luscher T.F., Ruschitzka F., Enseleit F. Prognostic value of longterm blood pressure changes in patients with chronic heart failure. Eur J Heart Fail. 2017;19(7):837-842. https://doi:10.1002/ejhf.805. Epub 2017 Mar 27.

45. Massie B.M., Carson P.E., McMurray J.J., Komajda M., McKelvie R., Zile M.R., Anderson S., Donovan M., Iverson E., Staiger C., Ptaszynska A.; I-PRESERVE Investigators. Irbesartan in patients with heart failure and preserved ejection fraction. $N$ Engl J Med. 2008;359:2456-2467. https:// doi:10.1056/NEJMoa0805450.

46. Apostolos Tsimploulis, MD, Phillip H. Lam, MD, Cherinne Arundel, MD Steven N. Singh, MD, Charity J. Morgan, PhD, Charles Faselis, MD, 1,4 Prakash Deedwania, MD,1,6 Javed Butler, MD, MPH, MBA, Wilbert S. Aronow, MD, Clyde W. Yancy, MD, MSc, Gregg C. Fonarow, MD, and Ali Ahmed, MD, MPH Systolic Blood Pressure and Outcomes in Patients With Heart Failure With Preserved Ejection Fraction. JAMA Cardiol 2018;3(4):288-297. https://doi:10.1001/jamacardio.2017.5365.

Поступила 03.04.2019

Принята в печать 29.04.2019 